\title{
NUMERICAL SOLUTION OF INVISCID FLOW AROUND 3D AERIAL VEHICLES
}

\author{
EBRAHIMI* A., MAZAHERI ${ }^{* *}$ K. and KHATIBIRAD*** S.
}

\begin{abstract}
Fundamental steps for inviscid computational fluid dynamics (CFD) analysis of three dimensional aerial configurations including modeling and surface grid generation, volume grid generation and solution of the governing equations are studied. For modeling and surface grid generation a simple and rapid algorithm has been used, in which the body is divided into several pieces and each piece is defined with linear triangular patches. These patches are mapped to a 2D space and then the surface grid will be generated in the same space. Finally the inverse mapping to the physical space is obtained and appropriate data structure for 3D grid generation is generated. For volume grid generation, the Delaunay's triangulation method is used to generate an unstructured tetrahedral grid. Using Roe's upwind and the AUSM ${ }^{+}$ schemes the Euler equations are solved by using an explicit time marching algorithm. This algorithm is applied on several aerial configurations. Convergence and accuracy of the scheme is studied by different means. A few examples show that the method is applicable on a wide range of problems.
\end{abstract}

\section{KEYWORDS}

euler equations, triangular surface grid, unstructured tetrahedral grid

\section{INTROUDUCTION}

Computational fluid dynamics is now well developed and has been regarded as an alternative or complement to wind tunnel testing especially for elementary design. A lot of efforts have been devoted to the grid generation and solution methods. The desire to compute flows over complex configuration has spawned a surge of activity in the area of unstructured grids [1]. Unstructured grid is stronger in covering complex fields, automation of the process is simpler, variation of element size in grid is applicable and adaptation process is also simpler. There are a variety of unstructured grid generation methods. The two main approach of unstructured grid generation are Delaunay methods [2, 3, 4], and advancing Front methods [3, 5, 6]. In the Delaunay triangulation an existing mesh will be modified by introducing new nodes.

* Gratuate student, Dpt. of Aero. Eng., Sharif University of Tech., Tehran, Iran.

** Professor, Dpt. of Aero. Eng., Sharif University of Tech., Tehran, Iran.

*** Gratuate student, Dpt. of Aero. Eng., Sharif University of Tech., Tehran, Iran. 
In this scheme the initial nodes are connected to each other geometrically, which builds up a topologically credible grid. In Advancing Front methods, after description of the boundaries, a primary front will be formed. Then new nodes are introduced, which form a front and this front progress toward the outer domain. The grid generation algorithms initially need to a surface grid. Surface grid generation is one of the important prerequirement of the 3D grid generation. A bad surface grid can affect the next CFD calculations. For surface grid generation we need to an analytical definition of the surface. Geometrical modeling provides this mathematical definition. After the modeling, a convenient algorithm used to generate a surface grid. This grid is used as an input to the volume grid generation algorithm. Here for surface grid generation Khatibirad's algorithm [7] and for volume grid generation method developed by Bodaghabadi [8] is used. Further modification on 3D grid generation is done by Eynian [9]. For flow solution the upwind Roe's scheme applied. This method doesn't prediction always positive pressure and density, then a positivity conservation scheme called $\mathrm{AUSM}^{+}$is used.

\section{GEOMETRICAL MODELING AND SURFACE GRID GENERATION}

In this section our ultimate goal is to discretize a given surface. To discretize a complex body surface, two steps should be followed. The first step is to determine the location of suitable grid points on the surface, and the second is to define cells and neighborhood information. For the first step we need an analytic definition of the surface and for the second we need a procedure to define the connectivities. If we have a convenient mapping of the surface to a $2 \mathrm{D}$ parametric space, the task of grid generation and neighborhood definition is a trivial task. The grid points distribution and the grid configuration in the parametric space is achieved in a manner that guarantees acceptable grid quality in the physical space. Mapping of these results back to the physical space is also a trivial operation. In geometric modeling, we use two different methods for definition of a patch. For patches with curvature in one direction we use planar strip triangles, and for patches with curvature in two directions, we use square patches. Therefore, we first try to divide the whole surface to some patches with curvature in only one direction. For regions which this could not be done with acceptable accuracy, we use square patches with curvature in two directions. The grid generation is performed in the parametric space; therefore it may include "bad" cells in the physical space, especially when the surface curvature is too much. Two local procedures, "edge swapping" and "smoothing", will improve the grid quality without any change in the number of grid points.

\section{Overall Modelling Algorithm}

The algorithm in step-by-step manner is described here for some patches with curvature in one direction:

1. The whole body surface is divided to many patches, so that each patch corresponds to a part of the surface with curvature mostly in one direction. Number of patches is a user-defined parameter.

2. Each patch is divided to many planar strip triangles, selected normal to the surface curvature, Fig.1.

3. Boundary grid points are generated on the boundary of each patch.

4. Intersections of patches are examined, and based on user-defined parameters intersection boundary points are generated. 
5. Each patch is mapped into a planar two-dimensional space which is called the computational space, Fig.2. Here we keep information about our basic triangles, which are used later for mapping of grid cells back to the physical surface.

6. An automatic grid generator is used for Delaunay grid generation in this space. Fig. 3 shows the grid in the parametric plane. A grid-smoothing algorithm is called, if necessary.

7. Finally the generated cells with the corresponding neighborhood information are mapped back to the physical body surface. Because the patches are convex, in the physical space each cell is checked with its neighborhoods and if this configuration does not satisfy the surface curvature, diagonal swapping is done. Fig.4 shows the grid in the physical space.

Many standard methods are used for modeling and grid generation of surfaces with curvature in different directions [10], and here we have used the standard sixteen points modeling.

\section{VOLUME GRID GENERATION}

A combination of advancing-front type point insertion and Delaunay criteria in cell connections is used for volumetric tetrahedral grid generation [3]. The basic steps are described in the [11] and [8]. Finally a Laplacian smoothing is used to improve the grid quality [12]. In this way, relatively high quality elements are generated that are used by Finite Volume algorithms in Euler equations solutions.

\section{SOLUTION SCHEME}

The first order time integration of Euler equations on each control volume and explicit discretization produces:

$$
\underline{Q}^{n+1}=\underline{Q}^{n}-\frac{\Delta t}{V} \sum_{i=1}^{\text {nface }} \underline{F}_{n}^{i} S_{i}
$$

where $\underline{Q}$ is the vector of conservative properties and $\underline{F}_{n}^{i}$ is the normal flux across face $i . S_{i}$ is the face area, $V$ is the cell volume and $\Delta t$ is the local time step. Superscripts $n$ and $n+1$ denote the current and the next time step. A cell-centered method, in which tetrahedral grid elements are used as control volumes, is used. The fluxes are discretized by two different methods, Roe's approximation Reimann solver [13] and Liou's AUSM ${ }^{+}$scheme [14].

\section{Roe's Scheme}

The Godonov-type uses the solution of Reimann problem for flux approximation. The Roe's scheme is the most famous algorithm in this family and it is powerful in numerical simulation of fluid flow with shock waves and other gas dynamic features. The Roe's flux function is:

$$
\underline{F}_{\kappa}=\frac{1}{2}\left[\underline{F}\left(\underline{Q}_{L}\right)+\underline{F}\left(\underline{Q}_{R}\right)-|\underline{\underline{A}}|\left(\underline{Q}_{R}-\underline{Q}_{L}\right)\right]_{\kappa}
$$

where $\mathrm{R}, \mathrm{L}$ represent the right and left state of the face, respectively [13]. Matrix $\underline{\underline{A}}$ is Jacobian of fluxes that is computed with Roe averaged quantities, which is introduced in several references with details [15]. 


\section{Liou's Scheme}

Euler codes often fail because either the density or the pressure becomes negative in some cell. This happens in situations where a high Mach number flow expands around a corner. Small negative values may approximate the true solution within the truncation error of the scheme, but are nevertheless unacceptable [16]. Einfeldt et al. [17] introduced the term 'positively conservative' to refer to a conservative scheme that would, given physically meaningful data, predict positivity density and pressure for all time. For one-dimensional flows they proved that the Godunov scheme [18] is positively conservative, but any Godunov-type scheme based on a linearized Riemann problem, for example Roe's scheme [13], does not have this property. There are several first order schemes qualified to be positively conservative in one space dimension. Among them Liou's reported $\mathrm{AUSM}^{+}$scheme [14]. AUSM is a hybrid scheme that combine the efficiency of FVS and the accuracy of FDS. This scheme first developed by Liou and Steffen [19] and further extended to low-Mach-number applications by Edwards and Liou [20]. AUSM is a class of flux splitting scheme that is remarkably simple and accurate, yielding vanishing numerical diffusivity at the stagnation. The result is competitive with flux-difference splitting schemes in term of accuracy but having a lower per-equation cost [21]. Also the scheme has no matrix operation and thus is more efficient. Unlike Roe's splitting, the scheme dose not involves differentiation of fluxes. The construction of AUSM is based on the observation that the inviscid flux comprises from convective and pressure contributions for which the implementation of upwinding principles is carried out separately. The relationship of the scheme has been discussed more thoroughly in [22].

\section{RESULTS}

\section{Surface Grid Generation}

The presented scheme is capable to model many aerospace configurations. For example the surface mesh that is generated for a wing alone configuration has been showed in Fig.5. A classical wing-body configuration is modeled in Figures 6 to 7.This simple airplane is modeled as a combination of a body and a few wings. This surface grid has 5704 nodes and 11404 cells. In Fig. 8 a rocket configuration with three sets of wings is modeled.

\section{Volumetric Meshes}

A three-dimensional volume grid generator is used to generate the grid around three sample configurations. The first one is the grid around a space launch vehicle with a set of wings. This geometry consists of a cone, a cylindrical body and four wings with NACA0012 section. The far-field boundary is a cylindrical surface. A cross section of this grid is shown in Fig.9. The second sample is a space launch vehicle with three boosters, Fig.10. This example is a sample of the volumetric mesh around multi bodies. The third sample mesh is the grid around an airplane. This geometry and volume mesh is shown in Figures 11 to12.

\section{Validation}

Validation of different subschemes that are used here are discussed in their references but to validate the developed code some standard cases have been solved. Three examples are solved here. The first one is a wedge. Steady state 
solution of the supersonic flow with freestream Mach number 3 over the wedge with 18.43 degrees angle is considered. This solution has 22544 nodes and 121364 cells. The Mach contours and comparison of Mach distribution with the analytical solution across the shock is presented in Fig.13 From numerical solution the angle of the shock is 34.6 degrees, compared to 35.95 degrees in the exact solution.

The second sample is flow over a cone. Because of the symmetry, only one-half of the cone is considered. The far-field boundary is a cylindrical surface. Mach 2 flow over the cone with 11.3 degrees angle is simulated here. Grid generation for this case is performed with 5557 nodes and 24934 cells. Comparison of pressure ratio with the analytical solution in cross view is presented in Fig. 14 for $y=0.0$ section. With this comparison we can see that numerical solution can predict the location of shock wave very well.

The last case is the supersonic Mach 2 on a cone with 10 degrees half-angle with 10 degrees angle of attack. Pressure coefficient on the cone surface is compared with Shankar [23] in Fig.15. As expected, the pressure reaches to its minimum on the leeward side of the cone and then increases. In the current solution this minimum is detected almost accurately. Weak shock waves are not well developed on the leeward side, and strong windward shocks dominate the physics of this problem. Indeed a higher order scheme and adaptation can lead to better results in the solution.

\section{Aerodynamic Applications}

Creditability of the schemes used is shown in simulation of a complete airplane and space launch vehicle. To show the difference of the $\mathrm{AUSM}^{+}$scheme and the Roe's scheme, a space launch vehicle with four wings is considered. The freestream Mach number is 2.5 with zero angle of attack. Because of sudden expansion in the base of this geometry, the density or the pressure in some cells become negative for Roe's scheme. In such cases the characteristic values are improved (Harten correction). Mach contours for $\mathrm{AUSM}^{+}$scheme are shown in Fig.16.

To investigate the capability of these schemes for flow simulation of multi bodies, a space launch vehicle with three boosters is considered. In this example the interaction of shocks with different sources is captured. The freestream Mach number is 3.0 with zero angle of attack. The Mach contours solution on the surfaces of bodies with AUSM scheme, and the pressure contours in two $x$ and $z$ const. sections are shown in Fig.17.

Finally a complete airplane with wings and tails is considered. Freestream Mach number is 0.5 with zero angle of attack. Flow simulation is performed with $\mathrm{AUSM}^{+}$ scheme. The Mach contours around the airplane in two $y$ and $x$ const. sections are shown in Fig.18 The pressure contours on the surface of airplane are presented in Fig.19.

\section{CONCLUSIONS}

A simple and fairly fast algorithm for geometric modeling and surface grid generation of simple 3D configurations is presented. Popular tetrahedral unstructured Grid generation around this configuration is discussed and implemented. Inviscid flow simulation is performed with Roe and $\mathrm{AUSM}^{+}$scheme. Different applications are used to show robustness of the algorithm in three basic steps (3D modeling, 3D grid generation and solution). In future viscous effects will be added for more accurate simulation of the aerial vehicles. 


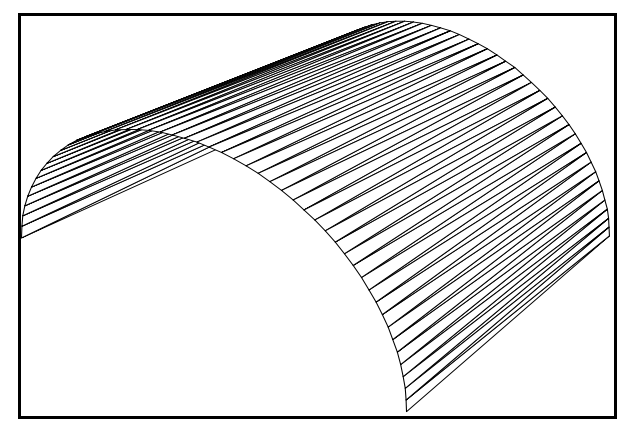

Fig.1. Planar strip triangles



Fig.3. Parametric plane

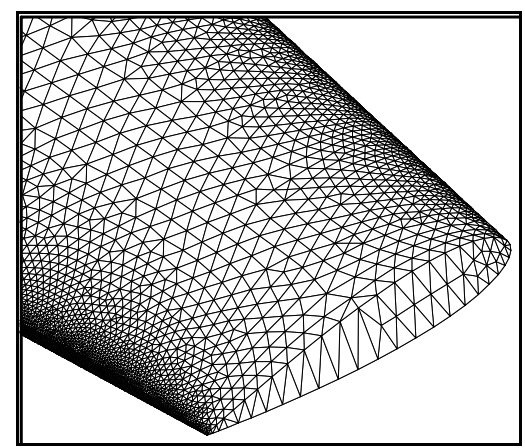

Fig.5. A wing

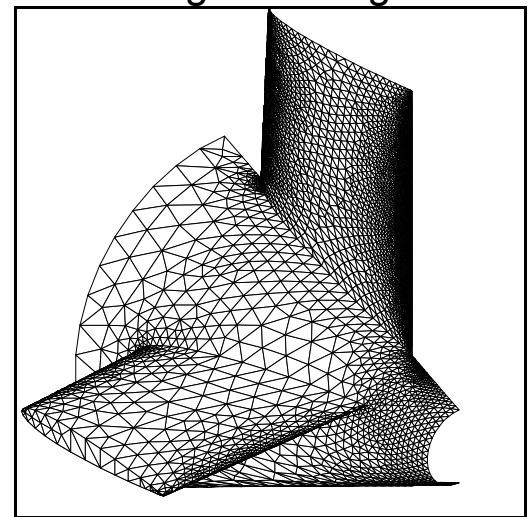

Fig.7. Some wing and body

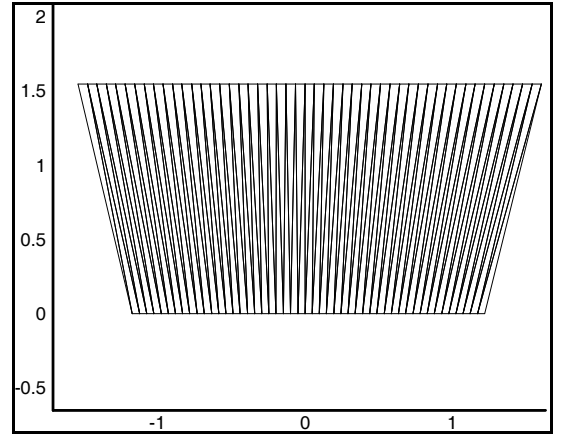

Fig.2. Mapping patch into a 2D space

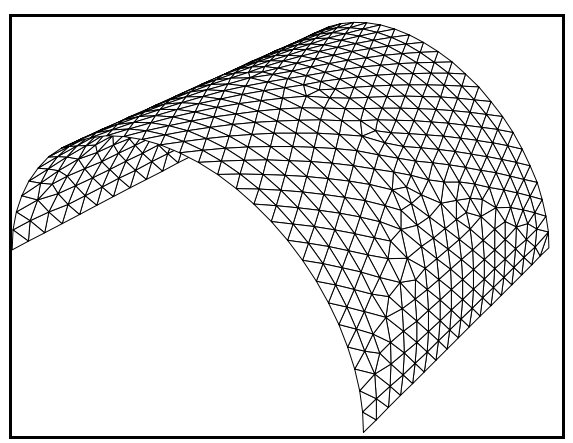

Fig.4. Physical space

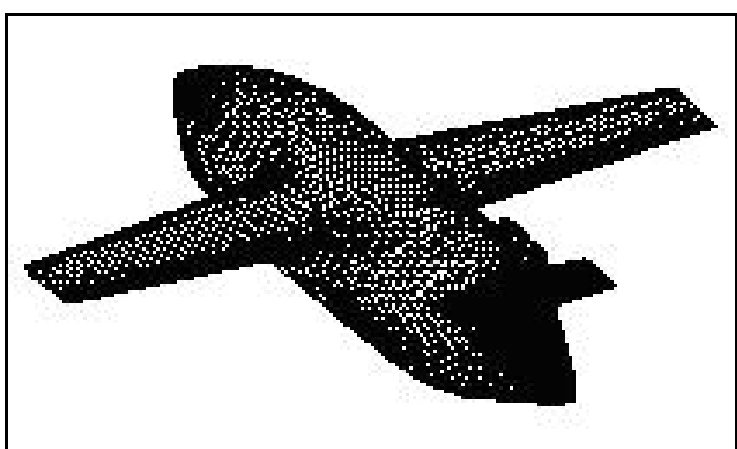

Fig.6. Simple model for airplane

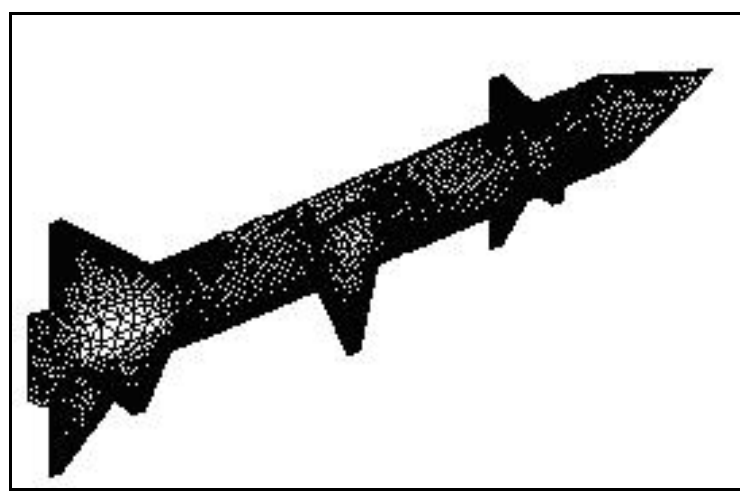

Fig.8. A rocket with three sets of wings 

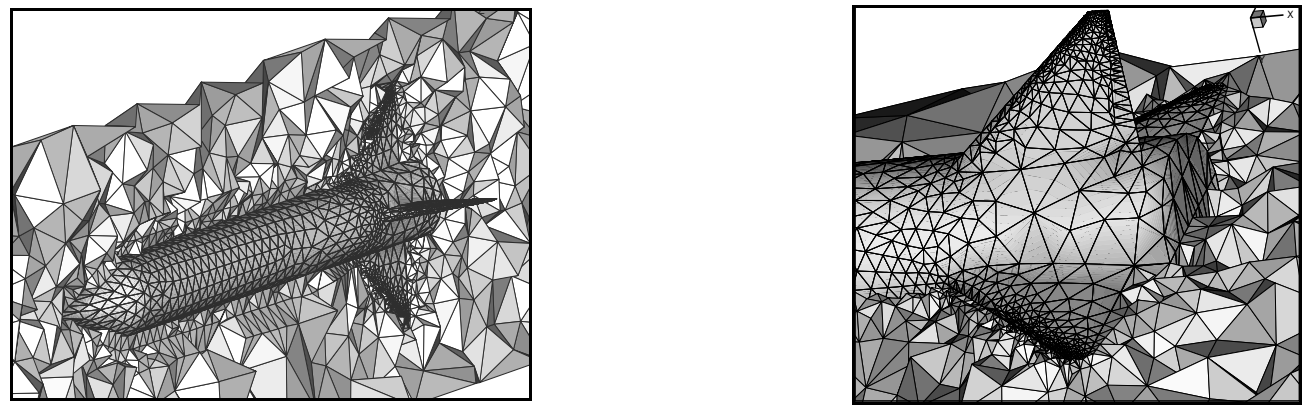

Fig.9. A section of volumetric grid around the rocket
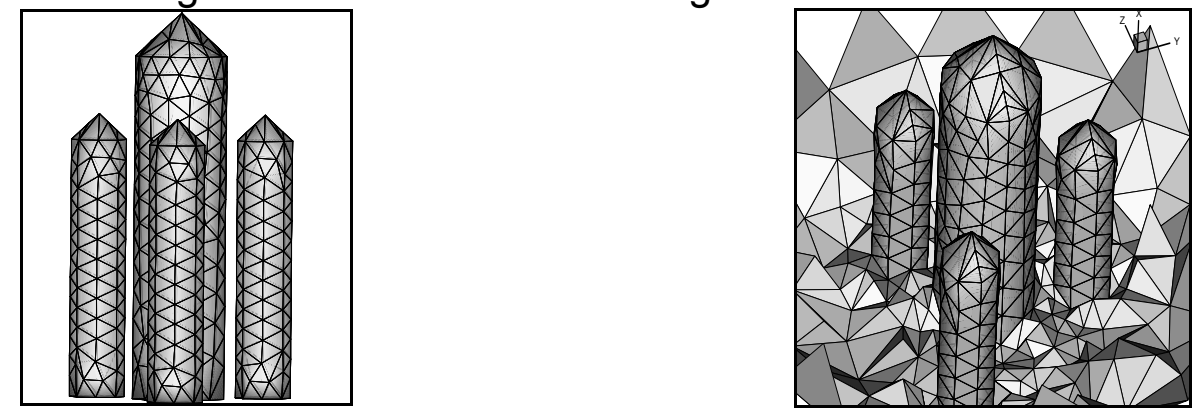

Fig.10. Surface grid and volumetric grid around a space launch vehicle

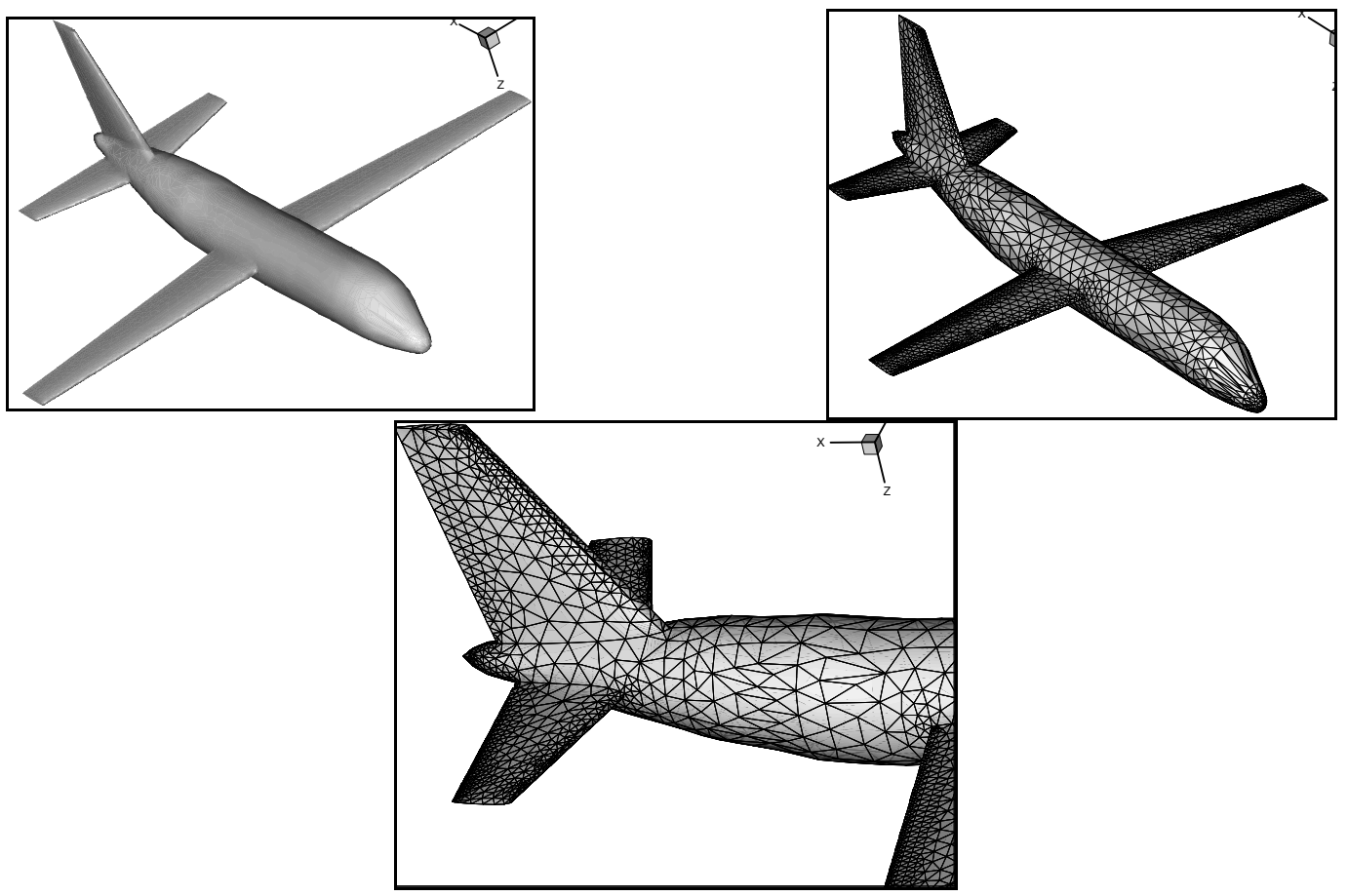

Fig.11. Geometry and the surface grid of an airplane 

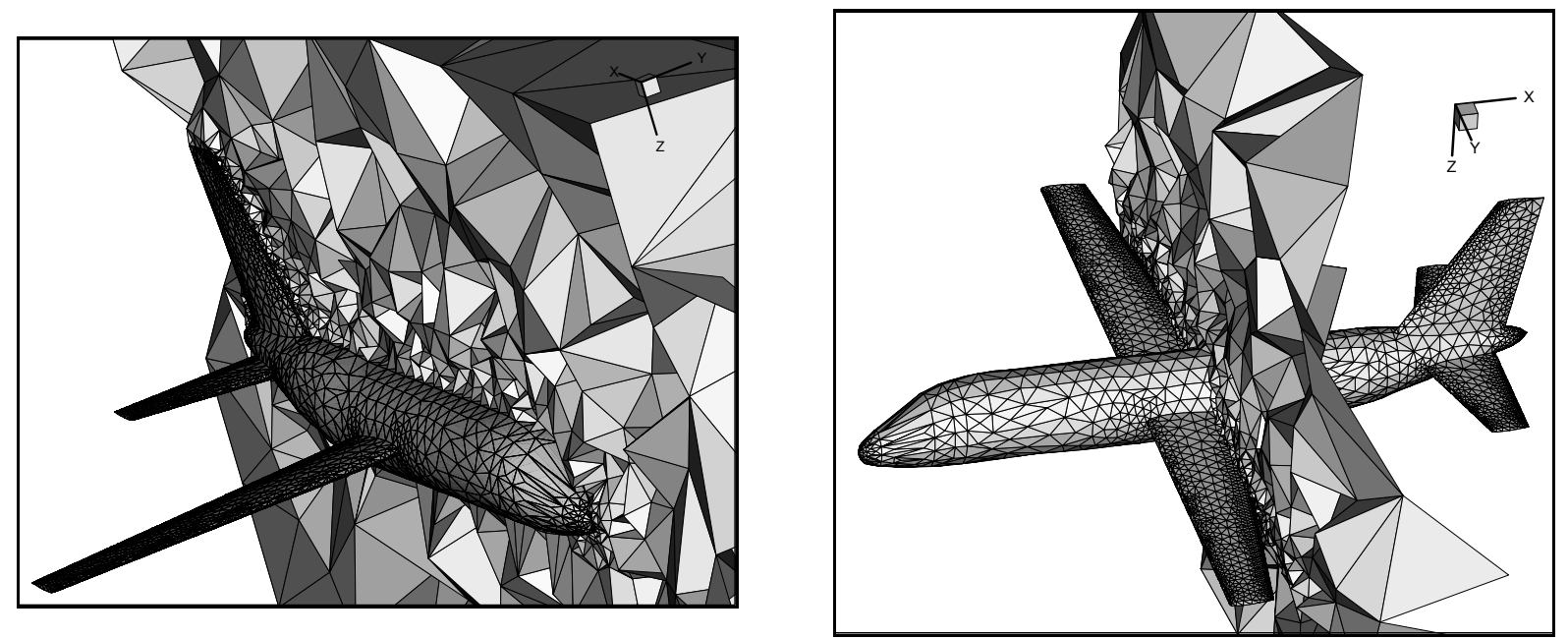

Fig.12. Some sections of volumetric grid around the airplane
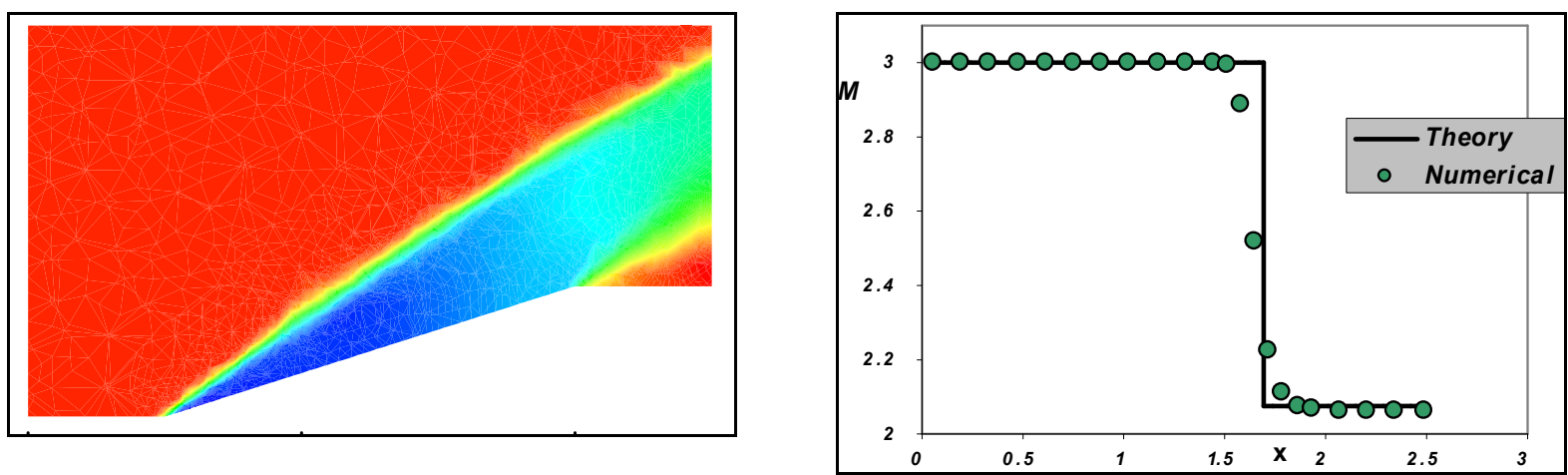

Fig.13. Mach contour and comparison the analytical with numerical solution

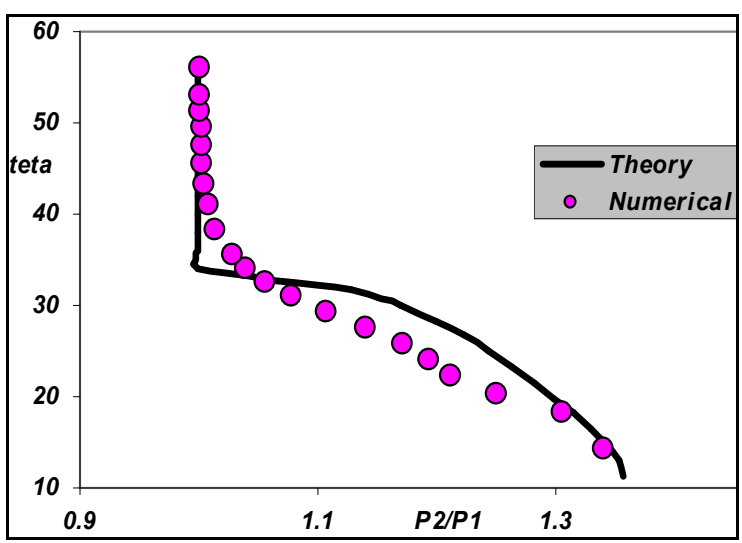

Fig.14. Comparison of the analytical with the numerical solution

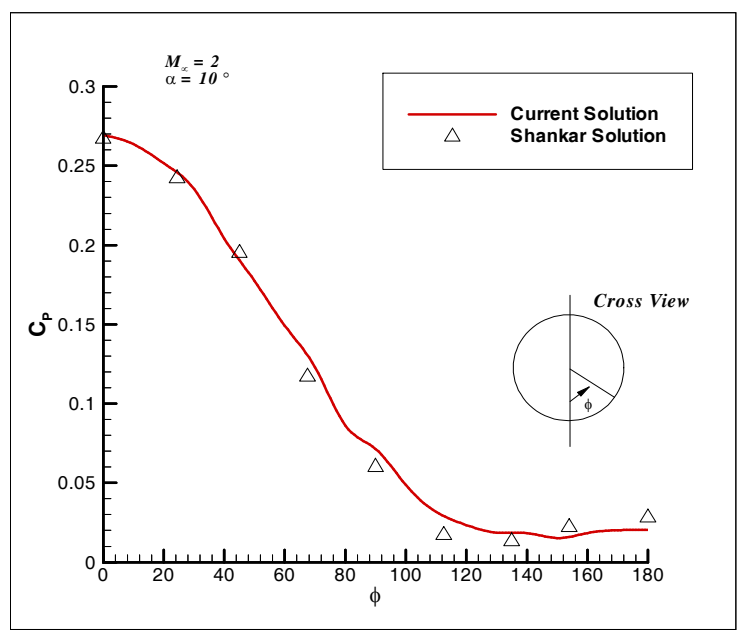

Fig.15. Circumferential pressure coefficient 


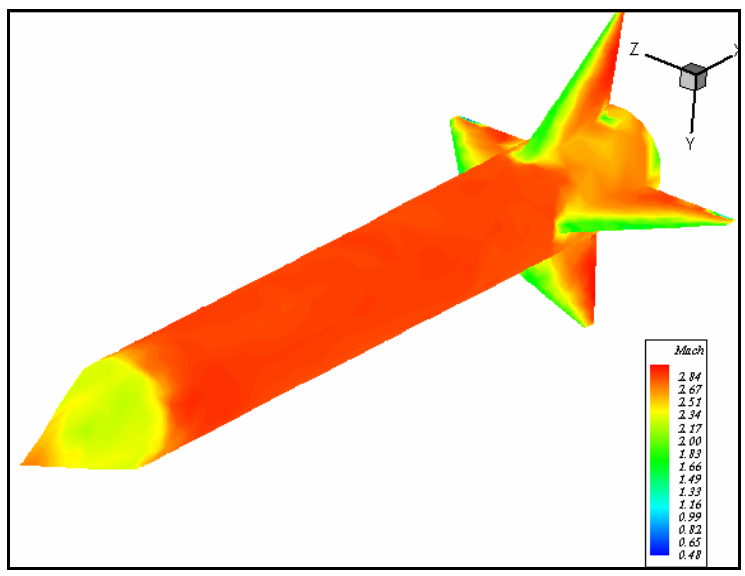

Fig.16. Mach contour
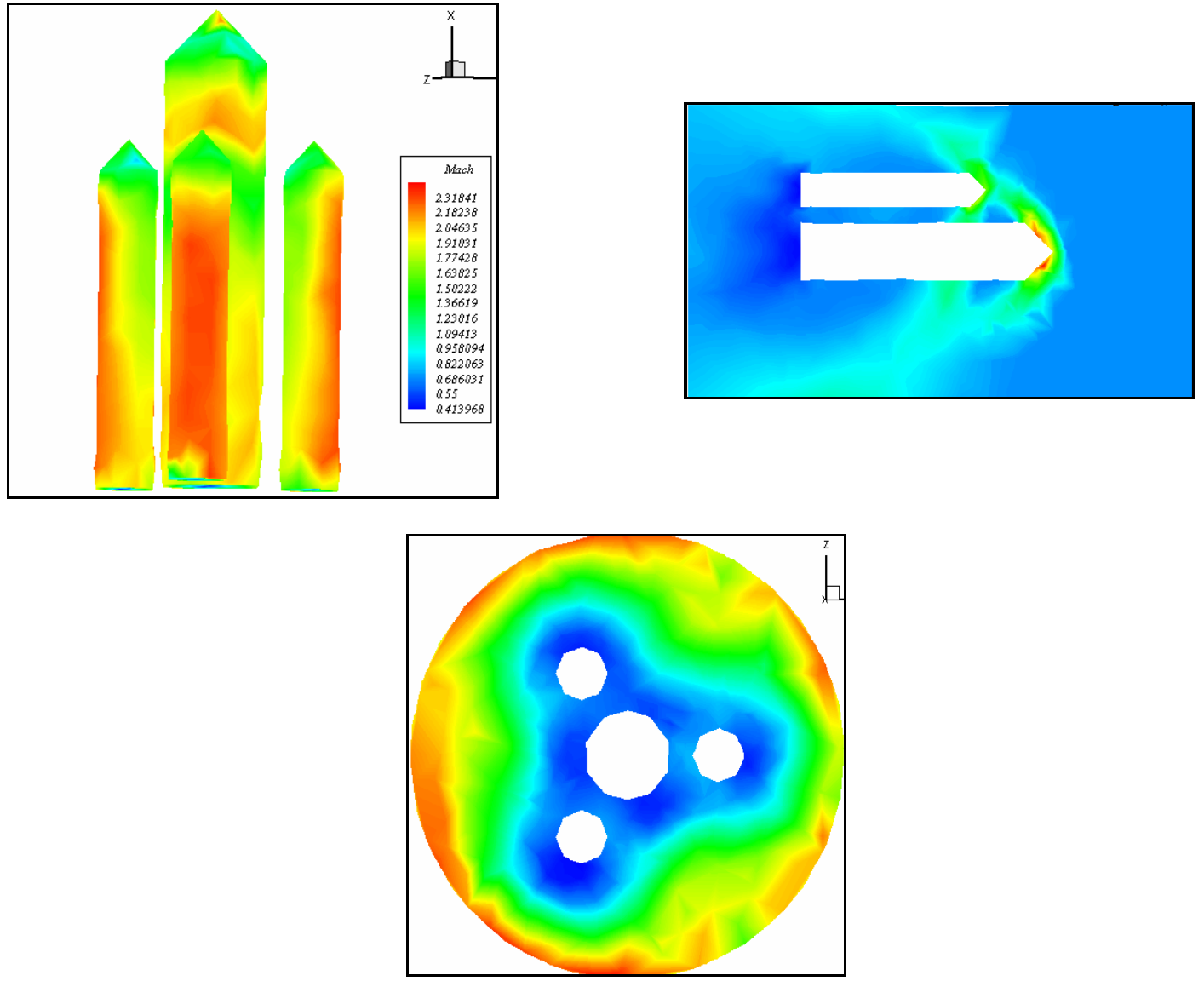

Fig.17. Surface Mach contours and contour of pressure in some sections 

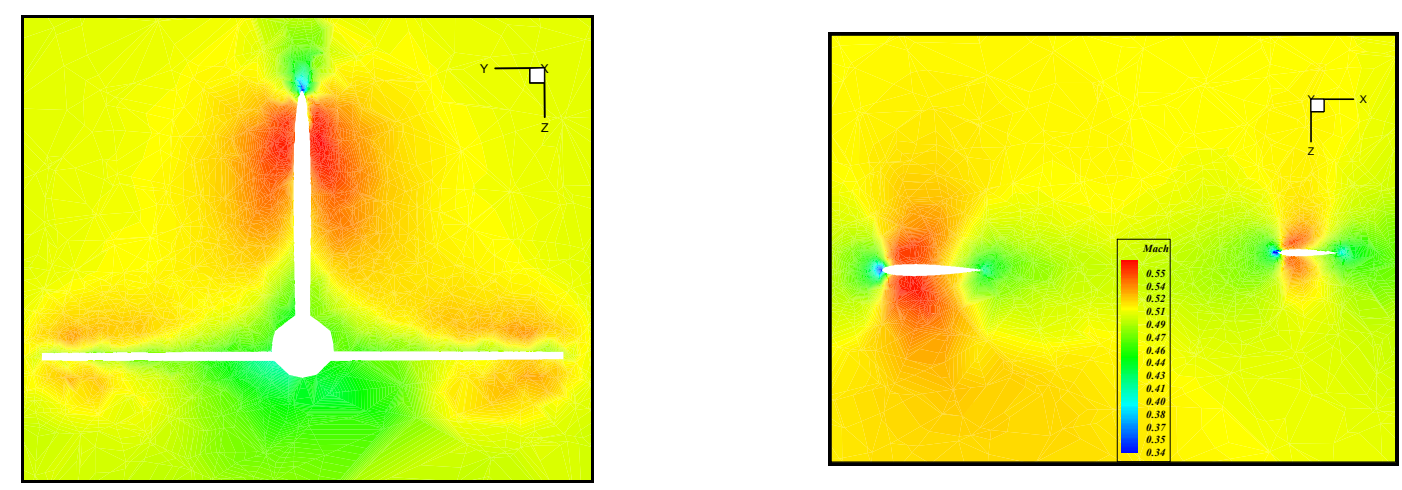

Fig.18. Mach contour in some sections



Fig. 19. Surface contour of pressure

\section{REFERENCES}

[1] Venkatakrishman, V., "Prespective on Unstructured Grid Flow Solvers", AIAA J., Vol. 34, No.3, pp 533-547, (1996).

[2] Barth, T.J., "On Unstructured Grids and Solvers", Von Karman Institute Lecture Series 1990-03 in Comp. Flu. Dyn., pp 1-65, (1990).

[3] Muller, J.D., Roe, P.L. and Deconinck, H., "A Frontal Approach for Internal Node Generation in Delaunay Triangulations", Int. J. for Num. Meth. in Flu., Vol. 17, No.3, pp 241-256, (1993).

[4] Rebay, S., "Efficient Unstructured Mesh Generation by Means of Delaunay Triangulation and Bowyer-Watson Algorithm", J. of Comp. Phy., Vol. 106, pp 125138, (1993).

[5] Marcum, D.L. and Weatherill, N.P., "Unstructured Grid Generation Using Iterative Point Insertion and Local Reconnection", AIAA J., Vol. 33, No.9, pp 1619-1625, (1995).

[6] Pirzadeh, Sh., "Three-Dimensional Unstructured Viscous Grids by the AdvancingLayers Method", AIAA J., Vol. 34, No.1, pp 43-49, (1996).

[7] Khatibirad, S., "Geometric Modelling of 3D Simple Configuration", M.S. Dissertation, Amir-Kabir University of Technology, (2001).

[8] Bodaghabadi, S., "Solution of the3D Inviscid Flow on the Adaptive Unstructured Grid”, M.S. Dissertation, Sharif University of Techology , (1998). 
[9] Eyniyan, M. and Mazaheri, K., "Grid Generation for Complicated Geometries", CFD2004 @nference , Canada, (2004).

[10] Mortenson, M.E., "Geometric Modelling", John Wiely \& Sons, Inc., (1985).

[11] Mazaheri, K. and Bodaghabadi, Sh., "Three-Dimensional Unstructured Grid Generation for Finite-Volume Solution of Euler Equations", Proc. of ICAS 2000 Cong., P247.1, Harrogate, UK, (2000).

[12] Hermann, L.R., "Laplacian-Isoparametric Grid Generation Scheme", J. of the Eng. Mech., ASCE, 102:749-756, (1976).

[13] Roe, P.L., "Approximate Reimann Solvers, Parameter Vectors, and Difference Schemes", J. ofComp . Phy., Vol. 43, pp 357-372, (1981).

[14] Liou, M., "A Sequel to AUSM: AUSM+", J. of Comp. Phy., Vol. 129, No.2, pp 364-382, (1996).

[15] Frink, N.T., Parikh P. and Pirzadeh S., "A Fast Upwind Solver for the Euler Equations on Three-Dimensional Unstructured Meshes", AIAA-91-0102, (1991).

[16] Linde, T. and Roe, P.L., "Robust Euler Codes", AIAA-97-2098, (1997).

[17] Einfeldt, B., Munz, C.D., Roe, P.L., and Sjogreen, B., "On Godunov-Type Methods near Low Densities", J. of Comp. Phy., Vol. 92, pp 273-295, (1991).

[18] Godunov, S.K., "A Difference Scheme for Numerical Computation of Discontinuous Solution of Hydrodynamic Equations", Mat. Sb., Vol. 47, No.3, pp 271-306 (in Russian), (1959).

[19] Liou, M.S., and Steffen, C.J., "A New Flux Splitting Scheme”, J. of Comp. Phy., Vol. 107, pp 23-39, (1993).

[20] Edwards, J., and Liou, M.S., "Low-Diffusion Flux Splitting Methods for Flows at All Speeds", AIAA J., Vol. 36, No.9, pp. 1610-1617, (1998).

[21] Shahbazi, M., "Analysis of 2D Hypersonic Real Flow on an Adaptive Unstructured Grid”, M.S. Dissertation, Sharif University of Technology, (1998).

[22] Liou, M.S., "Probing Numerical Fluxes: Mass Flux, Positivity, and EntropySatisfying Property", AIAA Paper 97-2035, (1997).

[23] Shankar V., "Treatment of Conical and Nonconical Supersonic Flows by an Implicit Marching Scheme Applied to the Full Potential Equation", Proc ASME/AIAA conf on Computers in Flow Predictions and Fluid Dyn Exp, Washington D.C., pp 163-170, (1981). 